\title{
Non-steroidal anti-inflammatory drug and aspirin use and the risk of head and neck cancer
}

\author{
J C Wilson ${ }^{*, 1}$, L J Murray ${ }^{1}$, C M Hughes ${ }^{2}$, A Black ${ }^{3}$ and L A Anderson ${ }^{1}$ \\ ${ }^{1}$ Centre for Public Health, School of Medicine Dentistry and Biomedical Sciences, Institute of Clinical Sciences, Block B, Queen's \\ University Belfast, RVH, Grosvenor Road, Belfast BT12 6BJ, UK; ${ }^{2}$ School of Pharmacy, Queen's University Belfast, Medical Biology \\ Centre, 97 Lisburn Road, Belfast BT9 7BL, UK and ${ }^{3}$ National Cancer Institute, Executive Plaza South, 6120 Executive Boulevard, \\ Rockville, MD 20852-7234, USA
}

Background: Evidence for non-steroidal anti-inflammatory drugs (NSAIDs) preventing head and neck cancer (HNC) is inconclusive; however, there is some suggestion that aspirin may exert a protective effect.

Methods: Using data from the United States National Cancer Institute Prostate, Lung, Colorectal and Ovarian Cancer Screening Trial, we examined the association between aspirin and ibuprofen use and HNC.

Results: Regular aspirin use was associated with a significant $22 \%$ reduction in HNC risk. No association was observed with regular ibuprofen use.

Conclusion: Aspirin may have potential as a chemopreventive agent for $\mathrm{HNC}$, but further investigation is warranted.

Over 600000 cases of head and neck cancer (HNC) occur annually (Freedman et al, 2007). Primary risk factors include tobacco use and excessive alcohol consumption (WHO, 2009). Head and neck cancer is associated with high levels of morbidity and mortality (Jayaprakask et al, 2006).

Several studies have shown non-steroidal anti-inflammatory drugs (NSAIDs) to reduce cancer risk (Rothwell et al, 2010, Bosetti et al, 2012). A systematic review by our group investigated the association between NSAID use and HNC (Wilson et al, 2011). Five studies met the inclusion criteria but each had significant limitations, including small sample size, lack of information on over-the-counter NSAID use and important confounding factors (Bosetti et al, 2003; Friis et al, 2003, 2006). Two studies indicated a protective effect of aspirin on HNC risk (Bosetti et al, 2003; Jayaprakash et al, 2006).

An upregulation of the expression of the inducible, inflammatory cyclo-oxygenase enzyme (COX-2) has been observed in many cancers, including HNC, and premalignant head and neck lesions (Mohan and Epstein, 2003; Altorki et al, 2004). It is thought
NSAIDs may prevent cancer development by inhibiting COX-2 and the downstream biological pathways implicated in carcinogenesis (Liao et al, 2007); other COX-2 independent pathways have also been suggested (Hwang et al, 2002: Kashfi, Ragas, 2005).

Using data from the National Cancer Institute Prostate, Lung, Colorectal and Ovarian Cancer (PLCO) randomised controlled screening trial a large scale prospective investigation of the effect of aspirin and ibuprofen on HNC risk was undertaken.

\section{MATERIALS AND METHODS}

The PLCO trial design is described in detail elsewhere (Prorok et al, 2000). Between November 1993 and September 2001, almost 155000 participants aged 55-74 years were recruited from ten US screening centres and randomly assigned to an intervention arm (screened for colorectal, lung, and ovarian or prostate cancer) or usual care arm. Over $99 \%$ of participants were followed up 
annually for cancer diagnosis and death. Cancer confirmation was ascertained from diagnostic information.

This investigation includes cases from both arms of the PLCO trial with no previous cancer history who completed the baseline questionnaire and provided information on aspirin and ibuprofen use. Participants were followed from enrolment to 31 December 2006.

Cases were defined as individuals diagnosed with a primary $\mathrm{HNC}$ at least 1 year after completion of the baseline questionnaire. Head and neck cancer sites comprised International Classification of Disease for Oncology version-2 topography codes C00.0-C14.8 and C30.0-C32.9. Lip cancers were excluded as the primary aetiology (exposure to UV light) was considered dissimilar to that of other included cancer sites.

Information on demographic characteristics, medical history, aspirin or ibuprofen use, anthropometric measures, and lifestyle was collected at baseline using a comprehensive self-completed questionnaire. Aspirin and ibuprofen use elicited a yes/no response to the following questions: during the last 12 months have you regularly used 'aspirin or aspirin-containing products such as Bayer, Bufferin, or Anacin', or 'ibuprofen containing products such as Advil, Nuprin or Motrin'. Regular use was not defined. Self-reported frequency of use was measured by the number of tablets usually taken: 1 per day, $\geqslant 2$ per day; 1 per week, 2 per week, 3-4 per week; $<2$ per month, $2-3$ per month, this was further categorised into daily and weekly/monthly use. Further information on frequency of use and dose usually taken (aspirin use only), was available in a supplementary questionnaire (SQX) completed between 2006 and 2007. Current body mass index (BMI) was calculated and categorised according to the World Health Organisation classification. Current smoking status was defined as never or ever use. Maximum cigarette pack years were calculated and categorised into quartiles. Self-reported alcohol consumption in the 12 months preceding interview was obtained at baseline for individuals in the screening arm.

Analysis. Using Cox proportional hazard modelling unadjusted and multi-variable adjusted hazard ratios (AHR) and 95\% confidence intervals (CI) for HNC risk were estimated. Participants were censored at the earliest of $\mathrm{HNC}$ diagnosis, death or 31 December 2006. Biologically plausible confounding variables were initially included in the model and excluded if $P$-values were $>0.10$. The possibility of aspirin users also using ibuprofen, and vice versa, was included as a possible confounder in the respective models. Body mass index values $<12$ and $>60 \mathrm{~kg} \mathrm{~m}^{-2}$ were excluded from the model as possible outliers using listwise deletion.

A subanalysis using participants with information on alcohol use was conducted. Adjusted hazard ratios were calculated with, and without, alcohol as a covariate to establish if alcohol use altered the risk estimate. Tests for interactions between aspirin/ ibuprofen use and smoking status and alcohol use were conducted. The effect of aspirin use on HNC risk was assessed by increasing alcohol use within those individuals in the screening arm who had information on alcohol use. Using available histological data a restricted analysis was conducted using only squamous cell carcinoma (SCC) HNCs.

Statistical analyses were conducted using STATA, version 11 (StataCorp, College Station, TX, USA). Tests of statistical significance were two-sided. $P$-values $<0.05$ were considered statistically significant.

\section{RESULTS}

After initial exclusions, 142034 individuals remained eligible for analysis. The mean follow-up time was $\sim 9$ years (range $0-13.1$ years), with a total person years of follow-up of 1276115 years. During follow-up, 316 individuals had a confirmed HNC. Cohort characteristics are shown in Table 1.

At baseline, $49.2 \%$ and $29.6 \%$ of participants reported regular aspirin and ibuprofen use, respectively. The later SQX provided an indication of aspirin strength; most non-HNC cases reporting daily usage in both questionnaires were taking low dose $(81 \mathrm{mg})$ aspirin. Those reporting weekly/monthly use in the SQX tended to take higher doses $(325 \mathrm{mg})$. A shift to increased frequency of use, particularly by those reporting weekly/monthly uses was also observed.

Table 1. Baseline Characteristics of participants within Study cohort (lip excluded)

\begin{tabular}{|l|c|c|c|}
\hline & $\begin{array}{c}\text { Total cohort } \\
(\boldsymbol{n}=142034)\end{array}$ & $\begin{array}{c}\text { HNC patients } \\
\text { Cho. }(\boldsymbol{n}=316)\end{array}$ & P-value \\
\hline Gender, $\boldsymbol{n}(\%)$ & $71643(50.4)$ & $244(77.2)$ & $<0.001$ \\
\hline Male & $70391(49.6)$ & $72(22.8)$ & \\
Female & $62.6(5.3)$ & $63.8(5.0)$ & $<0.001$ \\
Age (years) & &
\end{tabular}

Family history of HNC, $\boldsymbol{n}(\%)$

\begin{tabular}{|l|c|c|c|}
\hline Yes & $1738(1.2)$ & $8(2.5)$ & 0.034 \\
No & $140296(98.8)$ & $308(97.5)$ & \\
\hline
\end{tabular}

Race, $\boldsymbol{n}(\%)$

\begin{tabular}{|l|c|c|c|}
\hline White non-hispanic & $125532(88.4)$ & $279(88.3)$ & 0.739 \\
Black non-hispanic & $7286(5.1)$ & $14(4.4)$ & \\
Hispanic & $2684(1.9)$ & $9(2.9)$ & \\
Asian & $5345(3.8)$ & $11(3.5)$ & \\
Other & $1152(0.8)$ & $3(0.9)$ &
\end{tabular}

Education, $\boldsymbol{n}(\%)$

\begin{tabular}{|l|r|r|}
\hline 11 Years school & $10425(7.4)$ & $39(12.4)$ \\
12 Years/completed high & $32551(23.0)$ & $65(20.6)$ \\
$\begin{array}{l}\text { school } \\
\text { Post school/ some } \\
\text { college } \\
\text { College/postgraduate }\end{array}$ & $58715(34.4)$ & $111(35.2)$ \\
\hline
\end{tabular}

BMI $\left(\mathrm{kg} \mathrm{m}^{-2}\right)^{\mathrm{b}}, \mathrm{n}(\%)$

\begin{tabular}{|l|c|r|r|}
\hline$<18.4$ & $1138(0.8)$ & $5.1665(1.6)$ & $<0.004$ \\
$\geqslant 18.4$ to $<25$ & $45584(32.6)$ & $121(39.0)$ & \\
$\geqslant 25$ to $<30$ & $59457(42.5)$ & $132(42.6)$ & \\
$\geqslant 30$ & $33665(24.1)$ & $52(16.8)$ &
\end{tabular}

Smoking Status, $\mathbf{n}(\%)$

\begin{tabular}{|l|r|r|l|}
\hline Never & $65548(46.2)$ & $61(19.3)$ & $<0.001$ \\
Ever & $76450(53.8)$ & $255(80.7)$ & \\
\hline
\end{tabular}

Cigarette pack years max, $n(\%)$

\begin{tabular}{|l|r|r|r|}
\hline None & $65548(46.9)$ & $61(19.8)$ & \multirow{2}{*}{ > } \\
$>0-29$ & $37402(26.7)$ & $64(20.8)$ & $<001$ \\
$>29-49$ & $18533(13.3)$ & $63(20.4)$ & \\
$>49$ pack years & $18394(13.1)$ & $120(39.0)$ & \\
\hline Alcohol use, $\boldsymbol{n}(\%)$ & $10734(17.9)$ & $24(18.6)$ & 0.825 \\
\hline Never & $49367(82.1)$ & $105(81.4)$ & \\
Ever &
\end{tabular}

Abbreviations: $\mathrm{BMI}=$ body mass index; $\mathrm{HNC}=$ head and neck cancer.

${ }^{a}$ Continuous variables reported as mean \pm s.d.

$\mathbf{b}_{\text {Do not }}$ add up to 142034 due to exclusions of outliers $\left(12>\mathrm{BMl}>60 \mathrm{kgm}^{-2}\right.$ ) Only excluded in adjusted COX-analysis.

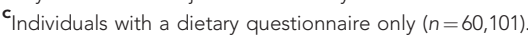


Unadjusted and multi-variable adjusted HRs for aspirin and ibuprofen use and HNC risk are shown in Table 2. After adjusting for possible confounders, a decreased risk of HNC was observed with regular aspirin use (AHR 0.78, 95\% CI 0.62-0.98). A significant reduction of HNC risk was observed between weekly and monthly aspirin use; daily aspirin use was not significantly associated with a reduced HNC risk, Table 2. No association was observed between ibuprofen use and HNC. When analyses were restricted SCC sites estimates for regular aspirin and ibuprofen use were similar to all HNCs (AHR 0.72, 95\% CI 0.56-0.93; AHR 0.96, 95\% CI 0.71-1.29, respectively).

The subtype specific analyses showed a significant protective association between aspirin use and laryngeal cancer (AHR 0.67, 95\% CI 0.45-0.99). An inverse, but non-significant, association between aspirin use and cancers of the oropharynx, and 'other sites combined' was observed (Supplementary Data, Table 1).

Including alcohol use as a possible confounder, the AHRs did not alter for aspirin or ibuprofen use. A significant interaction $(P=0.004)$ between alcohol and aspirin was observed. Stratification by increasing alcohol use revealed an increasing reduction in $\mathrm{HNC}$ risk in aspirin users compared with non-users. No protective association was observed in non-alcohol users (Table 3). No interaction between aspirin/ibuprofen use and tobacco use was evident.

\section{DISCUSSION}

Consistent with other studies (Bosetti et al, 2003; Jayaprakash et al, 2006; Ahmadi et al, 2010), we report a significant reduction in $\mathrm{HNC}$ risk with aspirin use, with the strongest protective effect for laryngeal cancers. No association was observed between HNC and ibuprofen use.

No association with ibuprofen use was observed. Compared with aspirin use, ibuprofen use was more infrequent and taken by proportionately fewer individuals; the reduction in power may, therefore, explain the lack of association. In addition, our analysis revealed a slight, non-significant, reduction in HNC risk with increased frequency of ibuprofen use, suggesting a possible causal effect. Alternatively, aspirin may exert different COX-2 independent effects on reducing cancer compared with ibuprofen (Elwood et al, 2009). A greater reduction in HNC risk was observed with weekly/monthly aspirin use than daily use. The later SQX indicated an increase in dosage and frequency of use among weekly/monthly aspirin users, which may explain the absence of a protective trend with increased frequency of use.

There was a near negligible difference between risk estimates with alcohol included in the model. Cohort alcohol intake was relatively low, possibly explaining the lack of effect on the HRs. Interaction analyses suggested that alcohol may be modifying the effect of aspirin use on HNC risk. A subanalysis in individuals with information on alcohol use revealed an increasing reduction in HNC risk, albeit non-significant, with aspirin use among

Table 3. Risk of head and neck cancer in relation to aspirin use: stratified analysis by increasing alcohol use

\begin{tabular}{|l|c|c|c|}
\hline $\begin{array}{l}\text { Number of } \\
\text { alcoholic } \\
\text { beverages per } \\
\text { day }\end{array}$ & $\begin{array}{c}\text { HNC } \\
\text { patients } \\
\text { no. }(\boldsymbol{n})(\%)\end{array}$ & $\begin{array}{c}\text { Cohort no. } \\
(\boldsymbol{n})(\%)\end{array}$ & $\begin{array}{c}\text { Multivariate } \\
\text { adjusted HR } \\
\text { (95\% Cl) }\end{array}$ \\
\hline Non-drinker & $24(18.7)$ & $10618(17.8)$ & $1.52(0.65-3.51)$ \\
\hline$>0-2$ Drinks per day & $81(63.3)$ & $41493(69.8)$ & $0.67(0.43-1.04)$ \\
\hline$>2$ Drinks per day & $23(18.0)$ & $7317(12.4)$ & $0.42(0.17-1.00)$ \\
\hline
\end{tabular}

Abbreviations: $\mathrm{Cl}=$ confidence interval; $\mathrm{HR}=$ hazard ratio; $\mathrm{HNC}=$ head and neck cancer. ${ }^{a}$ Multivariate adjustments: age at baseline (years), gender, BMI $(<18.5,18.5-<25,25-<30$, $\geqslant 30 \mathrm{~kg} \mathrm{~m}^{-2}$ ), tobacco use (none, $>0-29,>29-49,>49$ maximum cigarette pack years): Reference group: non-aspirin use.

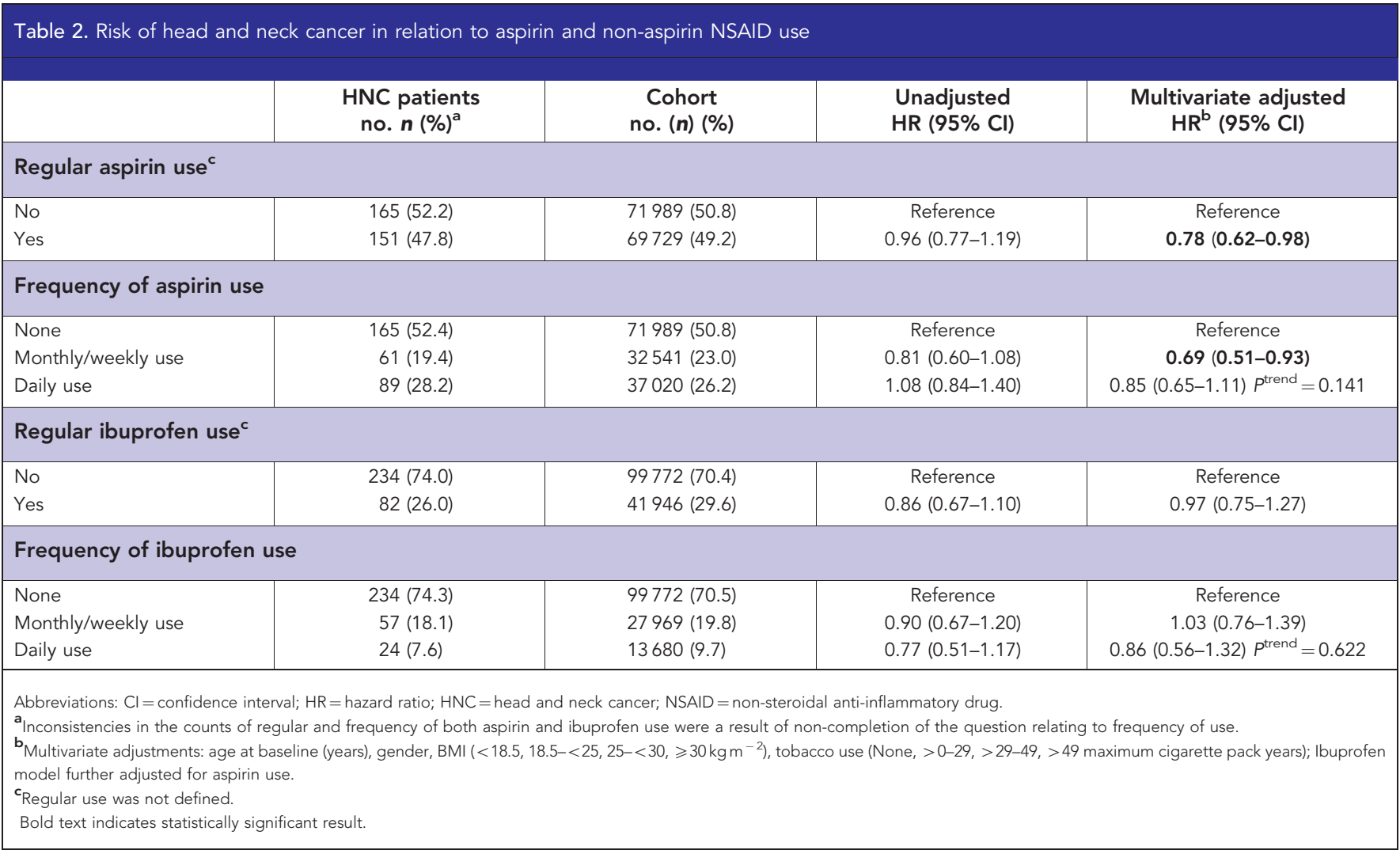


participants with increasing alcohol use. The exact mechanism by which this may be occurring is uncertain. Ethanol found in alcohol has been reported to act as a local irritant (Llewellyn et al, 2001) potentially leading to localised inflammation, which may possibly explain the observed reduction in HNC in aspirin users who consume alcohol. Previous studies have not reported alcohol as a possible effect modifier, further investigation is merited.

The site-specific analysis observed a significant protective effect with aspirin use and laryngeal cancer. The AHR was reduced for cancers of the oropharynx; however, the association was not significant (AHR 0.70 95\% CI 0.41-1.19), possibly due to the small numbers involved. Jayaprakash et al (2006) observed a similar reduction in oropharyngeal cancer risk with aspirin use. The majority of oropharyngeal sites have been described as human papillomavirus (HPV)-related (Ramqvist and Dalianis, 2010). There is some evidence to suggest an upregulation of COX-2 in HPV-infected tissues (Subbaramiah and Dannenberg, 2007).

Strengths of this study include the ability to control for important confounders, specifically tobacco use. Follow-up time was fairly long; follow-up exceeded $99 \%$. The study was limited by the lack of complete information on alcohol use, with information only available in the screening arm. However, there was little difference between participant characteristics within the two study arms and adjustment for alcohol had no effect on the point estimates. Dosage was from a later questionnaire and could only provide an indication of possible dosage.

This study supports the view that aspirin may have potential as a chemopreventive agent for HNC. There was little evidence to suggest that ibuprofen use was associated with reduced HNC risk. Further studies are required, in particular, those examining the strength and duration of aspirin use that may be required to exert a protective effect.

\section{ACKNOWLEDGEMENTS}

This research was supported by contracts from the Division of Cancer Prevention, National Cancer Institute; NIH, DHHS. We thank Drs Christine Berg and Philip Prorok, Division of Cancer Prevention, National Cancer Institute, the Screening Centre investigators and staff of the PLCO Cancer Screening Trial; Mr Tom Riley and staff, Information Management Services, Inc.; Ms Barbara O’Brien and staff, Westat, Inc. Most importantly, we acknowledge the study participants for their contributions to making this study possible.

\section{REFERENCES}

Ahmadi N, Goldman R, Seillier-Moiseiwitsch F, Noone A, Kosti O, Davidson BJ (2010) Decreased risk of squamous cell carcinoma of the head and neck in users of non-steroidal anti-inflammatory drugs. (published online). Int J Otolaryngol doi:10.1155/2010/424161.

Altorki HK, Subbaramaiah K, Dannenberg AJ (2004) COX-2 Inhibition in upper aerodigestive tract tumors. Semin Onco 31: 30-35.
Bosetti C, Rosato V, Gallus S, Cuzick J, La Vecchia C (2012) Aspirin and cancer risk: a quantitative review to 2011. Ann Oncol 23: 1403-1415.

Bosetti C, Talamini R, Franceschi S, Negri E, Garavello W, La Vecchia C (2003) Aspirin use and cancers of the upper aerodigestive tract. Br J Cancer 88: 672-674.

Elwood PC, Gallagher AM, Duthie GG, Mur LA, Morgan G (2009) Aspirin, salicylates, and cancer. Lancet 373: 1301-1309.

Freedman ND, Schatzkin A, Leitzmann MF, Hollenbeck AR, Abnet CC (2007) Alcohol and head and neck cancer risk in a prospective study. Br J Cancer 96: 1469-1474.

Friis S, Poulsen A, Pedersen L, Baron JA, Sorensen HT (2006) Use of nonsteroidal anti-inflammatory drugs and risk of oral cancer: a cohort study. Br J Cancer 95: 363-365.

Friis S, Sørensen HT, McLaughlin JK, Johnsen SP, Blot WJ, Olsen JH (2003) A population-based cohort study of the risk of colorectal and other cancers among users of low-dose aspirin. Br J Cancer 88: 684-688.

Hunter KD, Parkinson K, Harrison PR (2005) Profiling early head and neck cancer. Nat Rev Cancer 5: 127-135.

Hwang DH, Fung V, Dannenberg AJ (2002) National Cancer Institute workshop on chemopreventive properties of nonsteroidal antiinflammatory drugs: role of cox-dependent and -independent mechanisms. Neoplasia 4: 91-97.

Jayaprakash V, Rigual NR, Moysich KB, Loree TR, Nasca MA, Menezes RJ, Reid ME (2006) Chemoprevention of head and neck cancer with aspirin: a case-control study. Arch Otolaryngol Head Neck Surg 132: 1231-1236.

Kashfi K, Ragas B (2005) Cancer prevention: a new era beyond cyclooxygenase-2.J Pharmacol Exp Ther 314: 1-8.

Liao Z, Mason KA, Milas L (2007) Cyclo-oxygenase-2 and its inhibition in cancer. Is there a role? Drugs 67: 821-845.

Llewellyn CD, Johnson NW, Warnakulasuriya KAAS (2001) Risk factors for squamous cell carcinoma of the oral cavity in young people - a complete literature review. Oral Oncol 37: 401-418.

Mohan S, Epstein JB (2003) Carcinogenesis and cyclooxygenase: the potential role of COX-2 inhibition in upper aerodigestive tract cancer. Oral Oncol 39: $537-546$.

Pereg D, Lishner M (2005) Non-steroidal anti-inflammatory drugs for the prevention and treatment of cancer. J. Intern Med 258: 115-123.

Prorok PC, Andriole GL, Bresalier RS, Buys SS, Chia D, Crawford ED, Fogel R, Gelmann EP, Gilbert F, Hasson MA, Hayes RB, Johnson CC, Mandel JS, Oberman A, O’Brien B, Oken MM, Rafla S, Reding D, Rutt W, Weissfeld JL, Yokochi L, Gohagan JK. Prostate, Lung, Colorectal and Ovarian Cancer Screening Trial Project Team (2000) Design of the prostate, lung, colorectal and ovarian (PLCO) cancer screening trial. Control Clin Trials 21: 273S-309S.

Ramqvist T, Dalianis T (2010) Oropharyngeal cancer epidemic and human papillomavirus. Emerg Infect Dis 16: 1671-1677.

Rothwell PM, Wilson M, Elwin CE, Norrving B, Algra A, Warlow CP, Meade TW (2010) Long-term effect of aspirin on colorectal cancer incidence and mortality: 20-year follow-up of fi ve randomised trials. Lancet 376: 1741-1750.

Subbaramaiah K, Dannenberg AJ (2007) Cyclooxygenase-2 transcription is regulated by human papillomavirus 16 E6 and E7 oncoproteins: evidence of a corepressor/coactivator exchange. Cancer Res 67: 3976-3985.

Wang Z (2005) The role of COX-2 in oral cancer development, and chemoprevention/ treatment of oral cancer by selective COX-2 inhibitors. Curr Pharm Des 11: 1771-1777.

Wilson JC, Anderson LA, Murray LJ, Hughes CM (2011) Non-steroidal antiinflammatory drug and aspirin use and the risk of head and neck cancer: a systematic review. Cancer Causes Control 22: 803-810.

Supplementary Information accompanies this paper on British Journal of Cancer website (http://www.nature.com/bjc) 